\title{
Surface Morphology and Magnetic Properties of Isolated Cylindrical Nickel Nanowires
}

\author{
Musaab Salman Sultan ${ }^{1,2}$ \\ ${ }^{1}$ Information Security Department, Technical College of Informatics, Dohuk Polytechnic University, Kurdistan Region, Akre, Iraq \\ ${ }^{2}$ Accounting Department, Rawanduz Private Technical Institute, Kurdistan Region, Akre, Iraq \\ Email address: \\ dr.musaabs@gmail.com,dr.musaabs@yahoo.com
}

\section{To cite this article:}

Musaab Salman Sultan. Surface Morphology and Magnetic Properties of Isolated Cylindrical Nickel Nanowires. American Journal of Nanosciences. Vol. 3, No. 3, 2017, pp. 30-38. doi: 10.11648/j.ajn.20170303.11

Received: May 10, 2017; Accepted: June 5, 2017; Published: July 4, 2017

\begin{abstract}
In this article, the surface morphology and magnetic properties of isolated cylindrical nickel (Ni) nanowires fabricated by electrodeposition have been thoroughly investigated using scanning electron microscopy and Magneto-Optical Kerr Effect (MOKE) magnetometry, respectively. The surfaces of most nanowires were found to be homogenous, uniform, and cylindrical in shape. Some others show different diameters and surface features, including; protrusions and branches along their length. The diameter distribution of a wide range of nanowires was found to differ from their template pore diameters. These all variations are more likely due to defects exist in the internal surfaces of the pores within the template itself, or may be associated with the trapped air pockets within the pores during nanowires growth or due to the oxide formation or residual contaminants which may cover these wires. The nanowires lengths were found to differ from their actual lengths estimated during deposition growth. This was attributed to the breakage of nanowires into small sections during releasing process. The hysteresis loops obtained by applying a magnetic field at different angles with respect to the nanowires long axis showed square hysteresis loops with a sharp jump of Kerr signal during switching behaviour, as well as a high squareness ratio, indicating the dominance of shape anisotropy. These results are quite different from the measurements of high density templated nanowires reported in the literature, due to the small number of nearest neighbour nanowires, and hence no magneto-static interaction. The magnetisation reversal of such wires is well described by the non-uniform rotation of the curling model of domain reversal.
\end{abstract}

Keywords: Ferromagnetic Nanowires, Surface Morphology, Magnetic Properties, Magneto-Optical Kerr Effect (MOKE) Magnetometery, Magnetisation Reversal, Curling Model

\section{Introduction}

Cylindrical ferromagnetic nanowires are structures that have diameters in nanometer scale and unrestricted in length. Typical cylindrical nanowires exhibit high aspect ratios (length to diameter), and they can often be approximated as one dimensional materials [28-51]. These nanowires have many interesting properties that are not seen in a bulk or three dimensional structures. Therefore, fabrication and characterisation of such structures present interest from both fundamental and industrial points of view [14, 16, 28, 29, 37, $44,51,58]$. As an example; they may play a significant role in future applications, in particular; ultra-high density magnetic storage devices in hard disc drives and volatile magneto resistive random access memory (MRAM) [28-29].
Different techniques have been utilized to fabricate these structures, but electrodeposition has been proved to be simple, fast and a low cost technique, as well as using this technique, it is possible to produce different materials and multilayer as: thin films, nanowires or nanotubes $[5,8,11$, $12,14,18,31]$.

The magnetic properties of as-deposited two dimensional arrays of ferromagnetic nanowires have been investigated intensively over the last few decades using a wide range of characterising techniques [13-38]. These researches, however, have produced a significant insight into the physical behaviour of such arrays, where the magneto-static or the dipolar interactions among the wires have been found 
to dominate over the magnetic properties of an individual nanowire.

The intrinsic magnetic state of an individual or small number of closely packed ferromagnetic nanowires is a significant role for scientific point of view and demanding issue of industrial applications. Therefore, researchers were trying to investigate these properties by releasing such nanowires from their templates, and characterise them using micro SQIUD [57] or using further steps and various techniques to electrically connect them with the external circuitry, then using magneto-resistance setup to measure their magneto-transport properties [41-43, 54-55, 59]. Recently, within the development of computer systems and their memory, the Object Oriented Micro-Magnetic Framework (OOMMF) package has also been performed to investigate these properties using small dimensions of nanowires with simplified switching behaviour [21, 24, 36, 49].

Magneto-Optical Kerr Effect (MOKE) magnetometry has been widely used to study the magnetisation behaviour of two dimensional arrays of ferromagnetic nano and micro dots [10], stripes and planar nanowires [1, 7] fabricated by conventional electron beam lithography and lift-off techniques. In MOKE setup, however, the measured output of Kerr signal is proportional to the amount of magnetisation that depends upon the polarisation rotation of a linearly polarised laser light following its reflection from a magnetic surface $[1,7,10,32,45,5]$. Such MOKE measurements are potentially interesting, as it provides a very sensitive probe that is proportional to the change in the surface magnetisation to a depth of the order of skin-depth of the material [32]. Nevertheless, few articles have been published throughout this work discussing the magnetisation behaviour of $\mathrm{Ni}_{80} \mathrm{Fe}_{20}$ [38], $\mathrm{Ni}_{60} \mathrm{Fe}_{40}$ [50] and $\mathrm{Co}$ [45] nanowires using MOKE magnetometry.

Thus, the aim of the work presented here is to systematically investigate the surface morphology of a large number of template released cylindrical $\mathrm{Ni}$ nanowires deposited on oxidised silicon substrates from a dilute suspension, using a high-resolution scanning electron microscopy. Then, the magnetic properties of a closely packed two Ni nanowires with diameters of $\sim 300 \mathrm{~nm}$ and lengths of $\sim 8 \mu \mathrm{m}$ each have been measured using highly sensitive MOKE magnetometry in a longitudinal configuration at room temperature. The magnetisation reversal behaviour and the underlying physical mechanism are analyzed by applying a magnetic field at different angles with respect to the nanowires long axis. The results are then explained and compared with the literature and with the theoretical presentation of the curling model of domain reversal.

\section{Materials and Methods}

Two dimensional arrays of cylindrical ferromagnetic Ni nanowires were fabricated using electrodeposition technique. The fabrication process was carried out using an
Autolab-30 potentiostat and a conventional three electrode cell with $20 \mathrm{~cm}^{3}$ capacity. The electrolyte solution used was $0.57 \mathrm{M}$ of $\mathrm{NiSO}_{4}$ and $0.32 \mathrm{M}$ of $\mathrm{H}_{3} \mathrm{BO}_{3}$. The $\mathrm{pH}$ of the solution was maintained to approximately 3.5-4. An alumina template of thickness $\sim 60 \mu \mathrm{m}$ with a nominal pore diameter of $\sim 300 \mathrm{~nm}$ was used as a working electrode (cathode), after depositing a $100 \mathrm{~nm}$ pure gold layer $(99.99 \%),+$ on one side using thermal evaporation technique. The gold coated membranes are then used in the cell keeping the bare side of the template in front of the counter electrode. The voltage applied between the counter and reference electrodes was around $-0.85 \mathrm{~V}$ according to linear voltametry results. Once the electrodeposition pocess was completed, the contact wires were removed and the template washed in distilled water for the next step. The deposition time and the current density were chosen to produce nanowires with lengths of around $8 \mu \mathrm{m}$. More details on the fabrication process using electrodeposition can be found elsewhere [9, 27, 30, 35, 39].

The dissolution of the membrane and cleaning the wires before subsequent process is not an easy task. The objective is to achieve clean nanowires spread over the chips and sufficiently isolated from each other, to allow the magnetic measurements to be performed on an isolated nanowire. Nevertheless, this process was accomplished by dividing the templates into small parts with an approximate area of $1 \times 1$ $\mathrm{mm}^{2}$, then one or two pieces were placed in a small beaker containing two molars of sodium hydroxide $(\mathrm{NaOH})$, to dissolve the alumina membrane. The template was left in the solvent for a time of about 72 hours, by which the alumina template had dissolved and removed. With care, the $\mathrm{NaOH}$ solvent was drained out from the container and distilled water was added to wash the nanowires and remove the solvent. In order to be sure that $\mathrm{NaOH}$ solution was entirely removed from the beaker, this process was repeated for 4-5 times. However, care should be taken during this process that the released wires may fall out. Now, to remove the distilled water from the container, a small amount of isopropanol alcohol was added. To release the nanowires from their gold base substrate, the beaker was placed in an ultrasonic bath for a time of about 10 minutes. For further discussion on the template dissolving, nanowires releasing and deposition on silicon substrates can be found in reference [48]. Once the nanowires were released, a one drop was spilled onto silicon chips with a special micromarkers pre-fabricated by electron beam lithography. These markers were designed to be large enough to be seen in the microscope of MOKE system. After drying the solution, the wires are strongly stuck on the silicon chip surface. The position of the nanowires relative to the gold patterns was then carried out using scanning electron microscopy.

Imaging of nanowires and fabrication of gold markers on silicon substrates were performed using a high-resolution field emission scanning electron microscopy column on an FEI-Helios Nanolab dual beam FIB/SEM system with the electron beam energy of $\sim 10$ and $\sim 30 \mathrm{KeV}$, respectively. More details on the scanning electron microscopy, electron 
beam lithography and lift-off techniques are available in Refs $[8,22,23,56]$.

The magnetic analysis of isolated $\mathrm{Ni}$ nanowires was achieved using a highly sensitive MOKE magnetometry in the longitudinal configuration. In this system, the laser spot was focused to a diameter of approximately $5 \mu \mathrm{m}$. The change in the polarisation angle of the laser light after reflection from the sample was proportional to the longitudinal component of the wire magnetisation $[5,7,32$, 50]. The reflected light passed through an analysing prism and the intensity detected using a silicon photodiode connected to a high-bandwidth amplifier and a $\sim 1 \mathrm{GHz}-$ bandwidth oscilloscope. An AC electromagnet with a maximum field of \pm 450 Oe was used to switch the magnetisation of wires at a frequency of approximately 21 $\mathrm{Hz}$. To provide information about the angular dependence of the switching fields, the experimental setup allows for a rotation of the substrate around its surface normal by an angle up to $\sim 180$ degrees, in order to apply a magnetic field in the plane of the substrate at different angles with respect to the nanowires long axis. Thus, this setup was used for hysteresis loop measurements of a collection of closely packed two Ni nanowires. More information about MOKE setup can be found in Refs [5, 7, 32, 50].

\section{Results}

Figure 1 show high resolution scanning electron microscopy micrograph examples of single cylindrical $\mathrm{Ni}$ nanowires with different diameters, lengths and surface morphologies lying on the gold premarked silicon substrates following release and deposition from the same dilute suspension. Figure 2 shows the distribution histograms of (a) diameters, and (b) lengths of such dispersed individual $\mathrm{Ni}$ nanowires. The insets of figure 2 are scanning electron microscopy micrograph examples of the measured nanowires.

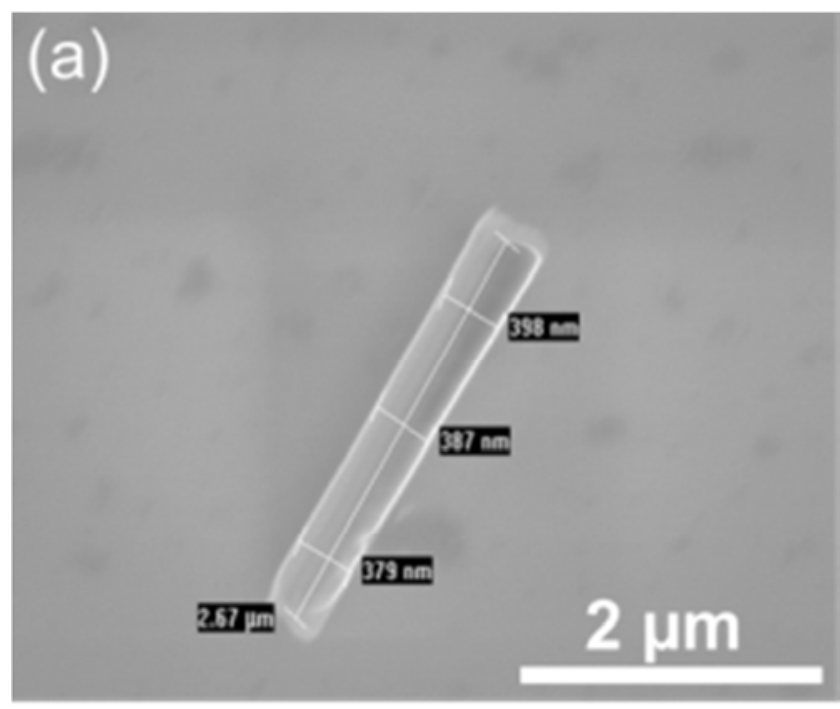

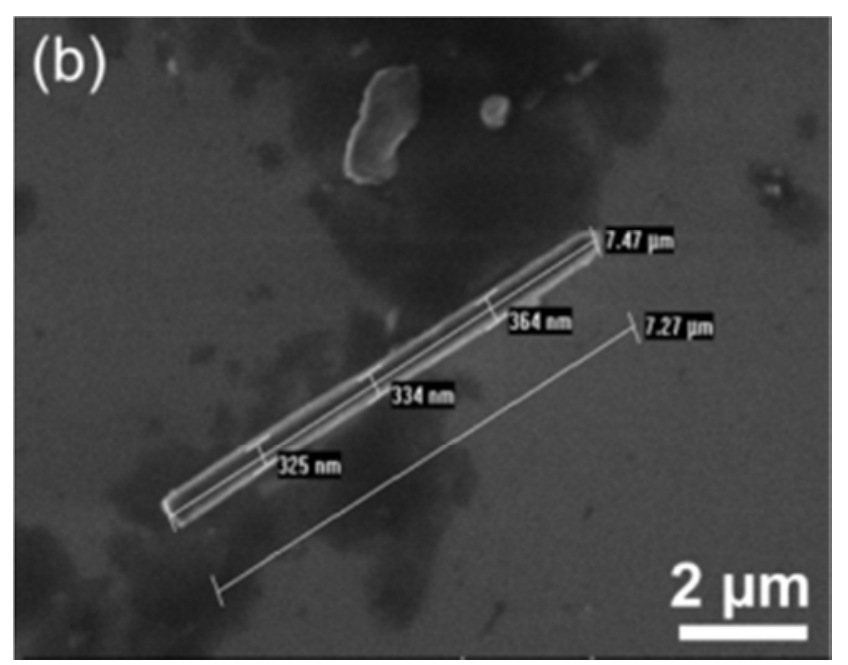
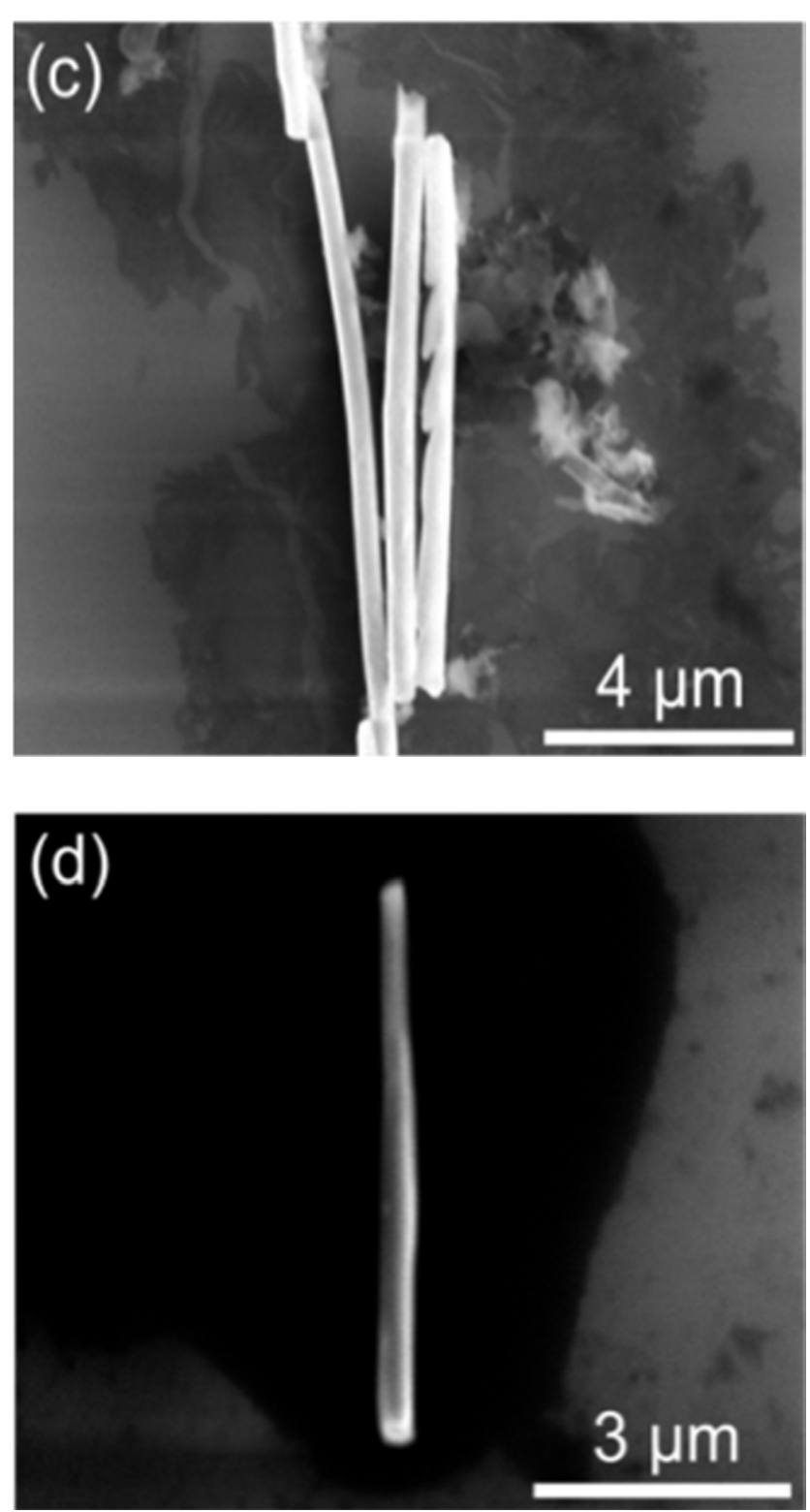

Figure 1. High resolution scanning electron microscopy micrograph examples of cylindrical Ni nanowires with different diameters, lengths and surface morphologies lying on the gold premarked silicon substrates following releasing and deposition from same dilute suspension. 

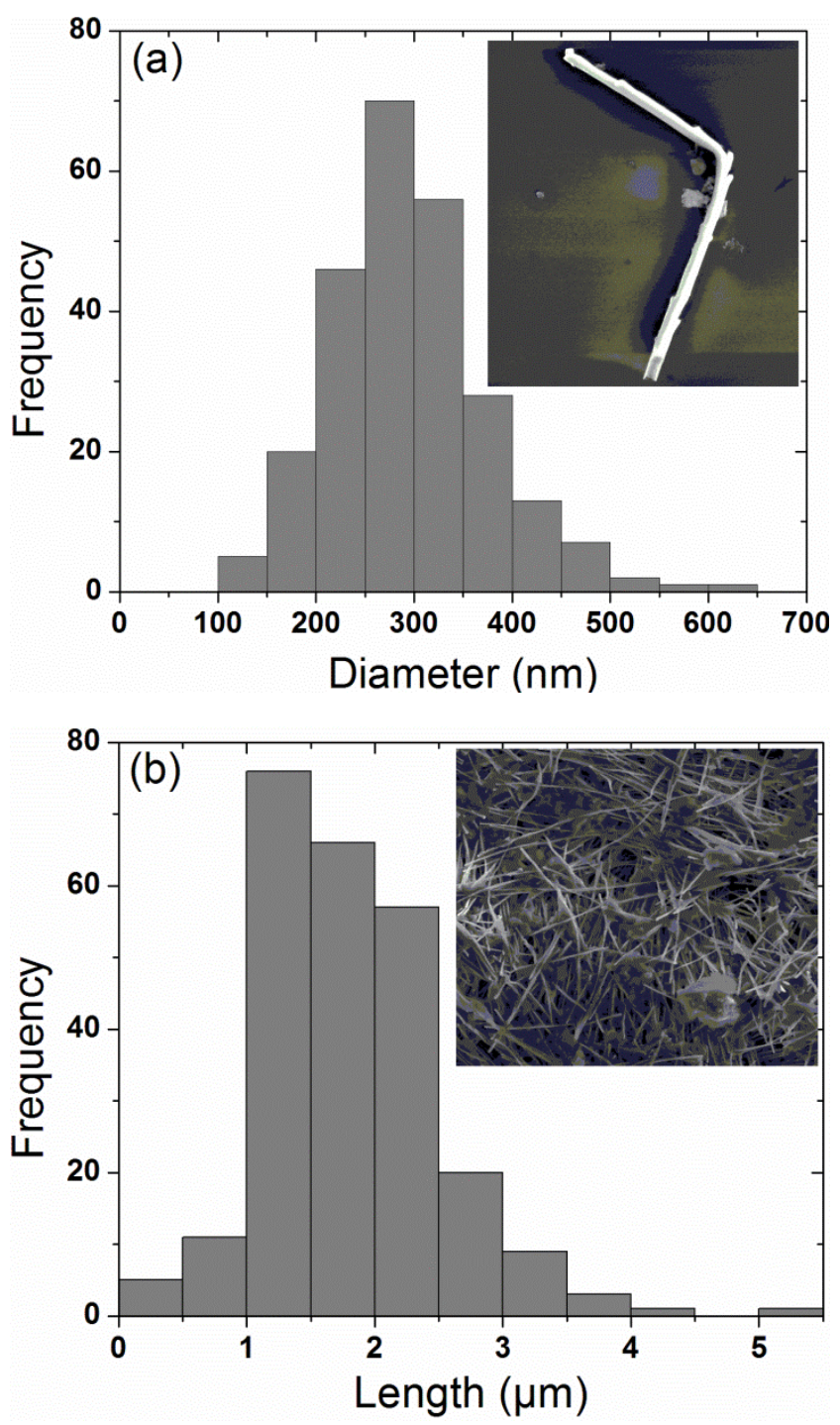

Figure 2. Distribution histograms of: (a) Diameters, and (b) Lengths of cylindrical Ni nanowires dispersed on silicon chips with nominal diameter and length of $\sim 300 \mathrm{~nm}$ and $\sim 8 \mu \mathrm{m}$, respectively. The figure insets are scanning electron micrograph examples of the measured nanowires.

During the course of MOKE measurements, it was difficult to get significant Kerr signals from such individual nanowires. Therefore, a collection of closely packed two cylindrical $\mathrm{Ni}$ nanowires with diameters of $\sim 300 \mathrm{~nm}$ and lengths of $\sim 8 \mu \mathrm{m}$ each was chosen for the magnetic measurements. Figure 3 shows scanning electron microscopy micrograph of the measured Ni nanowires. Even, with this closely packed nanowire, it was also a big challenge to detect a reasonable amount of Kerr signal, due to their small sizes and their curved surfaces which scatter the laser light in different directions.

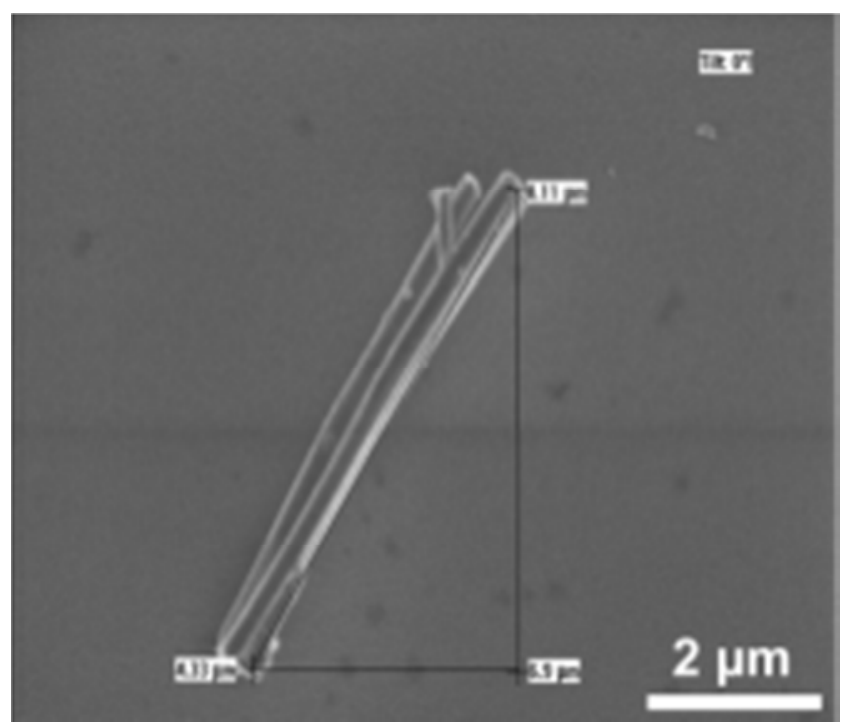

Figure 3. Scanning electron microscopy micrograph showing the collection of a closely packed two cylindrical Ni nanowires with $\sim 300 \mathrm{~nm}$ diameter and $\sim 8 \mu \mathrm{m}$ lengths each lying on the pre-marked silicon substrates.

Figure 4 show examples of the corresponding hysteresis loops obtained from this closely packed nanowires when the magnetic field was applied in four different angles $\left(0^{\circ}, 15^{\circ}\right.$, $30^{\circ}$, and $60^{\circ}$ ) with respect to the nanowires long axis. To get the coercivity distributions, the measurements were repeated in multiple locations on the same wires and for all the angles investigated here.

In all MOKE measurements, the signal to noise ratio of the hysteresis loops was found to be low. This is expected since the nanowires are very small compared to the laser spot size and the scattering effect. Importantly, the shapes of the hysteresis loops are quite different from those of two dimensional arrays of nanowires reported in the literature [6, $37,44,51]$. There is also, a change in the shape of the loop and a reduction in the coercivity as the external magnetic field is changed from parallel to the perpendicular direction.

The effect of changing the angle of applied magnetic field with respect to the nanowires long axis on the squareness ratio (the ratio of the remanent magnetisation to the saturation magnetisation) and the coercivity is plotted in Figure 5. The bars shown in Figure 5(b) are the distribution of the coercivity obtained from repeating measurements on the same wires at a given angle. High squareness ratios (about 0.86) were found in all the hysteresis loops. A reduction in the coercivity with increasing the nanowires angle with respect to the magnetic field applied. The average coercivity was found to be $\sim 110$ Oe and $\sim 75$ Oe when the magnetic field was applied at $0^{\circ}$ and $60^{\circ}$ to the nanowires long axis, respectively. 

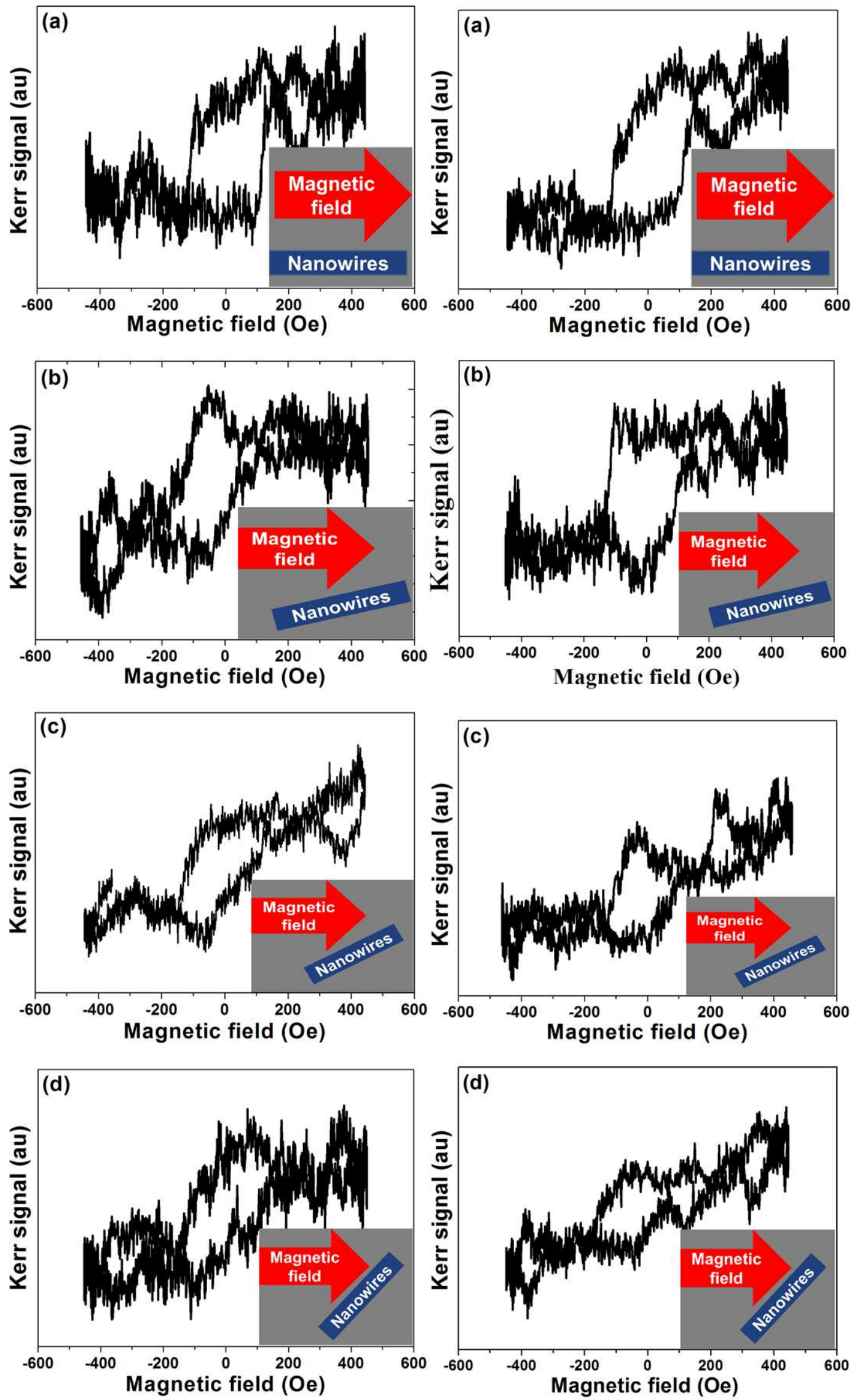

Figure 4. Hysteresis loop examples obtained from a collection of closely packed two Ni nanowires when the magnetic field was applied at four different angles: (a-a) $0^{\circ},(b-b) 15^{\circ},(c-c) 30^{\circ}$, and (d-d) $60^{\circ}$ with respect to the nanowires long axis. 

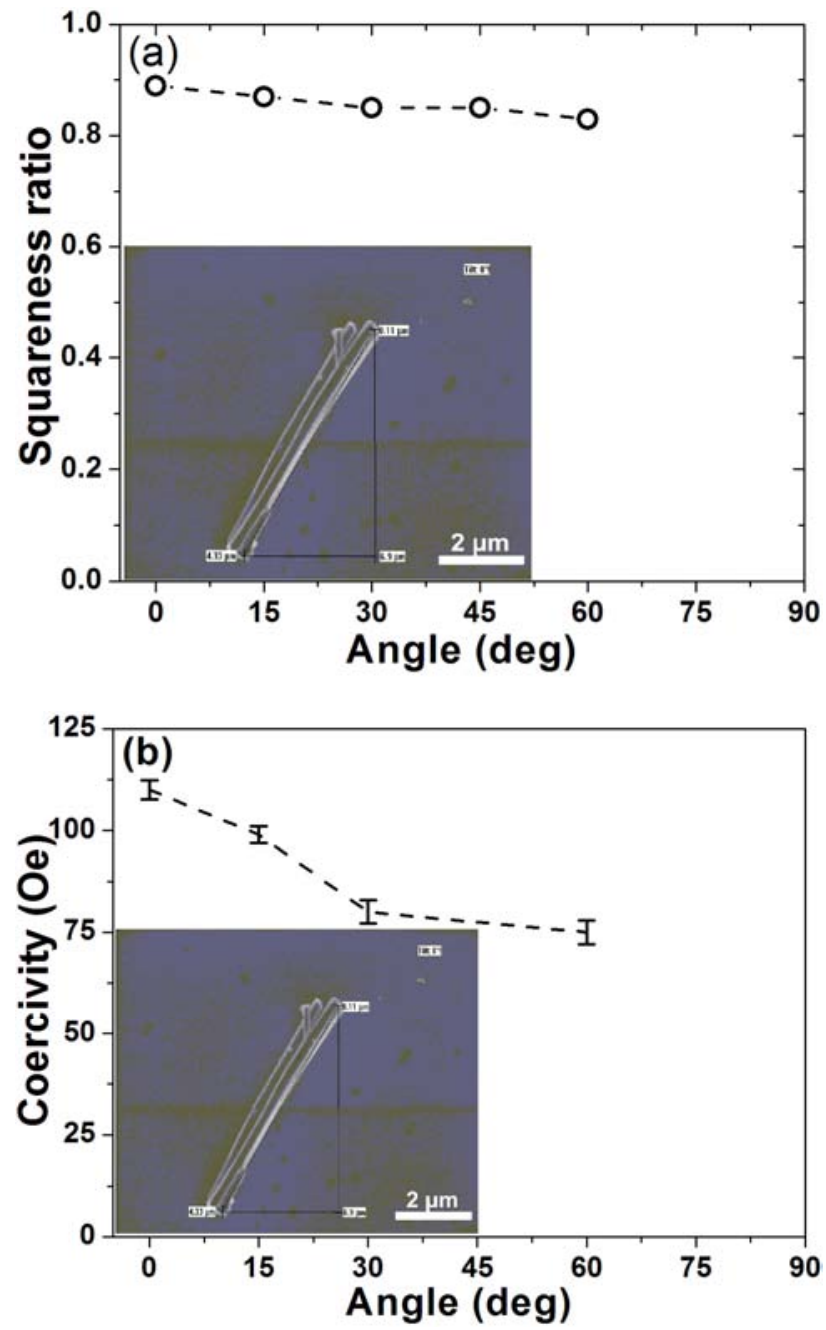

Figure 5. (a) Squareness ratio, and (b) Coercivity derived from the hysteresis loops of a closely packed two Ni nanowires at different angles of applied magnetic field with respect to the nanowire long axis. The dashed lines provide a guide to the eye.

\section{Discussion}

The surface morphology of a huge amount of individual cylindrical Ni nanowires spread over the silicon substrates was investigated using a high resolution scanning electron microscopy micrographs. The results demonstrate that these wires are smooth, uniform and cylindrical in shape. Some other wires showed a different diameter along their length $[17,40]$. Whilst others showed surface irregularities, including; protrusions and branches, as demonstrated in the micrograph examples shown in Figure 1. This variation in the surface morphology is more likely reflects the variation or defects exist in the internal surfaces of the pores within the template itself, or in some cases, may be associated with the trapped air pockets within the pores during nanowires growth $[26,40]$. Considerably, these defects may lead to changes in the actual magnetic properties of templated arrays of ferromagnetic nanowires. This is because, the surface roughness distort the spin-structure of ferromagnetic nanowires prior to the switching and it could lead to an increase or decrease in the switching field.

The high resolution scanning electron microscopy analysis, showed a wide range of diameters and lengths of nanowires dispersed on the silicon substrates. The distribution histograms were performed and are plotted in Figure 2. Clearly, a wide range of diameters $(100-650 \mathrm{~nm})$ and lengths $(1-5.5 \mu \mathrm{m})$ were observed. The nanowires diameter was found to differ from their nominal template pore diameter $(\sim 300 \mathrm{~nm})$, and this may be due to the oxide formation or residual templates and contaminants which may cover these wires or due to the variation in pore diameter themselves. Again, the variation in the nanowire diameters would make it difficult to entirely describe the magnetisation behaviour of two dimensional arrays of ferromagnetic nanowires.

The distribution of nanowires length was found to range from $\sim 0.5 \mu \mathrm{m}$ to $\sim 5.5 \mu \mathrm{m}$ which is far from their definite length estimated during deposition growth $(\sim 8 \mu \mathrm{m})$. This variation indicates that the sonication process used to separate nanowires from their base gold substrate causes the nanowires to break in different places. Hence, produce the wider distribution observed. Further analysis on the releasing process of ferromagnetic nanowires can be found in other published work $[48,50]$.

Despite of the difficulties arising from locating the nanowires within the MOKE illumination, the very small size of these wires with respect to laser spot size, and the rounded surfaces of these structures which leads to the scattering of reflected light into various directions, MOKE setup has been uninterruptedly applied for the measurements of hysteresis loops of a closely packed two Ni nanowires, so that it is not influenced by other nanowires at nearest neighbour. In templated nanowires, the interaction with other nanowires complicates the situation and makes it more complex to understand the exact magnetisation behaviour of a single or small number of closely packed nanowires.

The switching behaviour was found to be sharp in all angles of measurements in comparison with the hysteresis loops noticed in literature for two dimensional arrays of such Ni nanowires using other investigative techniques, including; vibrating sample magnetometer (VSM), superconducting quantum interference devices (SQUID) and alternating gradient magnetometers (AGM).

It is well known that the magnetic properties of an individual nanowire are governed by several anisotropies [ $[15$, 25], including; shape, magneto-crystalline, stress and surface anisotropies. The first anisotropy originates from the selfdemagnetising field due to it is cylindrical shape. The closely packed two Ni nanowires investigated here have the aspect ratio of about 27. The demagnetisation field, along the perpendicular direction to the nanowires long axis of such an aspect ratio can be approximated to be $2 \pi \mathrm{M}_{\mathrm{s}}=4346$ Oe [15], where $M_{s}$ is the saturation magnetisation of Ni bulk. This makes the long axial direction of the nanowires as the easy axis and its perpendicular direction as a hard axis of magnetisation by this amount of anisotropy field. The $\mathrm{Ni}$ 
nanowire is polycrystalline with a face centre cubic structure and it has a weak magneto-crystalline anisotropy (magnetocrystalline anisotropy constant $\mathrm{K}_{1} \approx 10^{4} \mathrm{~J} / \mathrm{m}^{3}$ ) [15]. Therefore, magneto-crystalline anisotropy of such nanowires can be neglected. Here, there is no chance to induce stress anisotropy to the nanowires, because the measurements were performed at room temperature and there is no source of stress anisotropy to introduce. Thus, stress anisotropy, can also be neglected from the analysis presented here. Lastly, the surface to volume ratio in such nanowires is low; therefore, the surface anisotropy makes a small contribution to the magnetisation behaviour of such wires due to uneven coordination number of the magnetic spins near the surface. These spins on the nanowires surface try to make the perpendicular direction as an easy axis of magnetisation. For more details on these mechansims can be found in Refs [15, 25, 44].

Thus, the closely packed two $\mathrm{Ni}$ nanowires investigated here behaves like a single nanowire and the resultant magnetic anisotropic direction is directed by the shape anisotropy of the nanowires. As a result, squared shaped hysteresis loops were obtained when the magnetic field was applied along their long axial direction. In contrary, the hysteresis loops for two dimensional arrays of Ni nanowires seen in literature showing the perpendicular direction as an easy axis of magnetisation $[6,37,44,51]$. This is solely due to the strong magneto-static interaction between the nearest nanowires, which makes the perpendicular direction of the templated nanowires is easy to magnetise. Here, the squareness ratio exhibits constant maximum values is expected due to the small number of nearest neighbors and hence no magneto-static interaction. The plot of coercivity against the angle of applied magnetic field shown in Figure 5 indicates that the coercivity decreases continuously with increasing nanowires angle due to a reduction in the shape anisotropy. Small distribution of the switching fields was obtained from repeating measurements at different locations on the same wires can be attributed to the variation in nanowire diameters or their misalignment with each other.

Various distinct mechanisms are able to describe the magnetisation reversal behaviour in nanoparticles, nanotubes and nanowires [2-4, 15, 46, 47]. For instance; coherent rotation of the Stoner - Wohlfarth model appears when the nanostructures are smaller than the exchange length, while curling and buckling modes occur when they are larger than the exchange length. In order to understand and explain the angular dependence of the coercivity presented here, it should first be noted that since the diameters of these nanowires $(\sim 300 \mathrm{~nm})$ are much larger than the exchange length, therefore one can expect a deviations of the micromagnetic structure away from the uniform rotation behaviour that is described by the Stoner-Wohlfarth reversal model [47]. Since buckling behaviour is expected to occur when the nanowire diameter is comparable to the exchange length (reduced radius around unity), buckling may also be excluded from the applicability here. The shape and aspect ratio of the closely packed two $\mathrm{Ni}$ nanowires investigated here is far from being a chain of spheres which would reverse as in the fanning model [15]. Therefore, the angular dependence of the switching fields is likely to be the consequence of an incoherent rotational process following the curling model of domain reversal.

\section{Conclusions}

Here, the surface morphology and magnetisation behaviour of electrodeposited isolated cylindrical $\mathrm{Ni}$ nanowires was intensively investigated using a high resolution scanning electron microscopy and MOKE magnetometry, respectively.

The surfaces of a wide range of $\mathrm{Ni}$ nanowires were found to be homogenous, uniform, and cylindrical in shape. Some other nanowires showed different diameters and surface features, including; protrusions and branches along their length. These variations were attributed to defects exist in the internal surfaces of the pores within the template itself, or due to trapped air pockets within the pores during nanowires growth. The diameters distribution histograms of a wide range of nanowires were found to differ from their template pores diameters. This was attributed to the oxide formation or residual templates and contaminants which may cover these wires or due to the defects in the template itself. The distribution of lengths was found to differ from their actual lengths estimated during deposition growth was attributed to the breakage of nanowires during the releasing process.

Due to the small surface area of nanowires, the signal to noise ratio of the hysteresis loops was found to be always low. The dipolar or magneto-static interaction between the $\mathrm{Ni}$ nanowires was expected to be low or even not exist, due to the small number of nearest neighbour, therefore the squareness ratio was found to be high (around 0.86) at all angles of measurements investigated here. Maximum coercivity was obtained (average $\sim 110$ Oe), when the magnetic field was applied parallel to the nanowires long axis, and low corecivity (average $\sim 75 \mathrm{Oe}$ ) observed when the magnetic field was applied perpendicular to the nanowires long axis and this was attributed to the reduction in the shape anisotropy. Small distribution of corecivity was obtained from repeating measurements at different locations on the same nanowires. This was attributed to the variation in nanowire diameters or their misalignment with each other. Furthermore, the angular dependence of corecivity was investigated regarding the mechanisms described in literature. The angular dependence of corecivity was likely to be the consequence of an incoherent rotational process following the curling model of domain reversal.

\section{References}

[1] Adeyeye, A. O., Bland, J. A. C., Daboo C., Lee J., Ebels U. \& Ahmed H. (1996) "Size dependence of the magnetoresistance in submicron FeNi wires" J. Appl. Phys., 79 (8), 6120.

[2] Aharoni, A. (1999) "Curling reversal mode in nonellipsoidal ferromagnetic particles" J. Appl. Phys., 86 (2), 1041. 
[3] Aharoni, A. (1986) "Magnetization buckling in a prolate spheroid" J. Appl. Phys., 60 (3), 1118.

[4] Aharoni, A. \& Shtrikman, S. (1958) "Magnetization curve of the infinite cylinder" Phys. Rev., 109 (5), 1522.

[5] Allwood, D. A, Xiong, G., Cooke, M. D. \& Cowburn, R. P. (2003) "Magneto-optical Kerr effect analysis of magnetic nanostructures" J. Phys. D: Appl. Phys., 36 (18), 2175.

[6] Aravamudhan, S., Singleton, J., Goddard, P. A. \& Bhansali, S. (2009) "Magnetic properties of Ni- Fe nanowire arrays: Effect of template material and deposition conditions" J. Phys. D: Appl. Phys., 42 (11), 115008.

[7] Atkinson D., Allwood D., Xiong G., Cooke, M. D., Faulkner C. C. \& Cowburn R. P. (2003) "Magnetic domain-wall dynamics in a submicrometre ferromagnetic structure" Nat. Mat., 2 (2), 85.

[8] Atkinson, D., Eastwood, D. S. \& Bogart, L. K. (2008) "Controlling domain wall pinning in planar nanowires by selecting domain wall type and its application in a memory concept" Appl. Phys. Lett., 92 (2), 022510.

[9] Bai, A. \& Hu, C.-C. (2003) "Iron-cobalt and iron-cobaltnickel nanowires deposited by means of cyclic voltammetry and pulse-reverse electroplating" Elec. Comm., 5 (1), 78.

[10] Bergenti I., Riminucci A., Arisi E., Murgia M., Cavallini M., Solzi M., Casoli F. \& Dediu V. (2007) "Magnetic properties of cobalt thin films deposited on soft organic layers" J. Mag. Mag. Mat., 316 (2), e987.

[11] Bryan, M. T., Atkinson, D. \& Allwood, D. A. (2006) "Multimode switching induced by a transverse field in planar magnetic nanowires" Appl. Phys. Lett., 88 (3), 032505.

[12] Cao, G. (2004) "Nanostructures and Nanomaterails Synthesis; Properties and Applications" Imperial College Press.

[13] Chen, W., Tang, S., Lu, M. \& Du, Y. (2003) "The magnetic properties and reversal of $\mathrm{Fe}-\mathrm{Co}$ nanowire arrays" J. Phys.: Condense Matter, 15 (26), 4623.

[14] Coey, J. M. D. \& Hinds, G. (2001) "Magnetic electrodeposition" J. Alloys and Compounds, 326 (1-2), 238.

[15] Cullity, B. D. \& Graham, C. D. (2009) "Introduction to magnetic materials" $2^{\text {nd }}$ edition, John Wiley and Sons Inc., Hoboken, New Jersey.

[16] Dao, N., Homer, S. R. \& Whittenburg, S. L. (1999) "Micromagnetics simulation of nanoshaped iron elements: Comparison with experiment" J. Appl. Phys., 86 (6), 3262.

[17] Daub, M., Enculescu, I., Neumann, R. \& Spohr, R. (2005) "Ni nanowires electrodeposited in single ion track templates" $J$. Opt. Adv. Mat., 7 (2), 865.

[18] Dumpich, G., Krome, T. P. \& Hausmanns, B. (2002) "Magnetoresistance of single Co nanowires" J. Mag. Mag. Mat., 248 (2), 241.

[19] Ferré, R., Ounadjela, K., George, J., Piraux, L. \& Dubois, S. (1997) "Magnetization processes in nickel and cobalt electrodeposited nanowires" Phys. Rev. B, 56 (21), 14066.

[20] Fert, A. \& Piraux, L. (1999) "Magnetic nanowires" J. Mag. Mag. Mat., 200, 338.

[21] Forster, H., Schrefl, T., Scholz, W., Suess, D., Tsiantos, V. \& Fidler, J. (2002) "Micromagnetic simulation of domain wall motion in magnetic nano-wires" J. Mag. Mag. Mat., 249 (1-2), 181.

[22] Golstein, J. I., Newbury, D. E., Echlin, P., Joy, D. C., Romig A. D. Jr., Lyman C. E., Fiori C. \& Lifshin E.(1992) "Scanning electron microscopy and X-ray" $2^{\text {nd }}$ edition, Plenum press, New Yourk \& London, P 177

[23] Goodhew, P. J., Humphrey, J. S \& Beanland, R. (2001) "Electron microscopy and analysis" $3^{\text {rd }}$ edition, Taylor \& Francis, London.

[24] Hertel, R. \& Kirschner, J. (2004) "Magnetization reversal dynamics in nickel nanowires" Physica B: Condensed Matter, 343 (1-4), 206.

[25] Jiles D. (1998) "Introduction to magnetism and magnetic materials" $2^{\text {nd }}$ edition, Chapman and Hall/CRC New York.

[26] Jin, C. G., Liu, W. F., Jia C., Xiang, X. Q., Cai, W. L., Yao, L. Z. \& Li, X. G. (2003) "High filling large area Ni nanowires arrays and the magnetic properties" J. Crystal Growth, 258, 337.

[27] Khan, H. R. \& Petrikowski, K. (2002) "Magnetic and structural properties of the electrochemically deposited arrays of Co and CoFe nanowires" J. Mag. Mag. Mat., 249 (3), 458.

[28] Kunz, A., Reiff, S. C., Priem, J. D. \& Rentsch, E. W. (2010) "Controlling individual domain walls in ferromagnetic nanowires for memory and sensor applications" International Conference on Electromagnetics in Advanced Applications, 248.

[29] Lee, S.-W., Jeong, M.-C., Myoung, J.-M., Chae, G.-S. \& Chung, I.-J. (2007) "Magnetic alignment of $\mathrm{ZnO}$ nanowires for optoelectronic device applications" Appl. Phys. Lett., 90 (13), 133115.

[30] Li, X. Wang, Y., Song, G., Peng, Z., Yu, Y., She, X. \& Li, J. (2009) "Synthesis and growth mechanism of Ni nanotubes and nanowires" Nano. Res. Lett., 4 (9), 1015.

[31] Lodder, J. C. (2004) "Methods for preparing patterned media for high-density recording" J. Mag. Mag. Mat., 272-276, 1692.

[32] Lupu N., Lostun M. \& Chiriac H. (2010) "Surface magnetization processes in soft magnetic nanowires" J. Appl. Phys., 107 (9), 09E315.

[33] McCord, M. A., and Rooks, M. J. (1997) "SPIE Handbook of Microlithography; Micromachining and Microfibrication" edited by P. Rai Choudhury (SPIE, Bellingham, WA), Vol. 1, link.aip.org/ link/APL/ 79/ 1721/ 1.

[34] Nalwa, H. S. (2002) "Handbook of Nanostructured Materials and Nanotechnology" Academic Press.

[35] Nasirpouri, F. (2007) "Template electrodeposition of magnetic nanowire arrays" Trans world Res. Net., 661, 37.

[36] Nian-mei, Han, Guang-hua, Guo, Guang-fu, Z., Wen-bing, S. \& Gao-fu, Men (2007) "Domain wall structure transition during magnetization reversal process in magnetic nanowires" Trans. Nonferrous Met. Soc. China, 17 (60571043), 1034.

[37] Nielsch K. \& Stadler, B. J. H. (2007) "Handbook of magnetism and advanced magnetic materials: Templatebased synthesis and characterization of high-density ferromagnetic nanowire arrays" Edited by Helmut Kronmuller and Stuart Parkin, Volume 4: Novel Materials, John Wiley \& Sons, Ltd. 
[38] Pignard, S., Goglio, G., Radulescu, A., Piraux, L., Dubois, S., Declémy, A. \& Duvail, J. L. (2000) "Study of the magnetization reversal in individual nickel nanowires" $J$. Appl. Phys., 87 (2), 824.

[39] Possin, E. George (1970) "A method for forming very small diameter wires" Rev. Sci. Ins., 41, 772.

[40] Rahman, I. Z., Razeeb, K. M., Kamruzzaman, Md., Serantoni, M. (2004) "Characterisation of electrodeposited Ni nanowires using NCA template" J. Mats. Proc. Tech., 153-154, 811.

[41] Rheem Y., Yoo B.-Y., Beyermann W. P. \& Myung N. V. (2007) "Electro-and magneto- transport properties of a single CoNi nanowire" Nanotech., 18 (12), 125204.

[42] Rheem Y., Yoo B.-Y., Koo B. K., Beyermann W. P. \& Myung N. V. (2007) "Synthesis and magnetotransport studies of single nickel-rich NiFe nanowire" J. Phys. D: Appl. Phys., 40 (23), 7267.

[43] Rheem Y., Yoo, B.-Y., Beyermann W. P. \& Myung N. V. (2007) "Magneto-transport studies of single ferromagnetic nanowire" Phys. Stat. Sol. (a), 204 (12), 4004.

[44] Sellmyer, D. J., Zheng, M. \& Skomski, R. (2001) "Magnetism of Fe, Co and Ni nanowires in self-assembled arrays" J. Phys.: Condens. Matter, 13, R433.

[45] Sharma S., Barman A., Sharma M., Shelford, L. R., Kruglyak, V. V. \& Hicken, R. J. (2009) "Structural and magnetic properties of electrodeposited cobalt nanowire arrays" Sol. St. Comm., 149, 1650.

[46] Skomski R., Zeng H., Zheng M. \& Sellmyer D. (2000) "Magnetic localization in transition- metal nanowires" Phys. Rev. B, 62 (6), 3900.

[47] Stoner, E. C. \& Wohlfarth, E. P. (1948) "A mechanism of magnetic hysteresis in heterogeneous alloys" Phil. Trans. $R$. Soc. Lond. A, 240 (826), 599.

[48] Sultan, M. S. (2017) "Fabrication and characterization of single electrodeposited fer romagnetic nanowires" Journal of Erbil Polytechnic University. To be published.
[49] Sultan, M. S., Atkinson, D. (2016) "Aspect-ratio dependence of magnetization reversal in cylindrical ferromagnetic nanowires" Material Research Express, 3, 056104.

[50] Sultan, M. S., Das B., Sen P., Mandal K. \& Atkinson D. (2012) "Template released ferromagnetic nanowires: Morphology and magnetic properties" J. Spintron. Magn. Nanomater., 1, 1.

[51] Sun L., Hao Y., Chien C. L. \& Searson P. C. (2005) "Tuning the properties of magnetic nanowires" IBM J. RES. \& DEV., 49 (1), 79.

[52] Vázquez, M. Pirota, K., Hernandez-Velez, M., Prida, V. M., Navas, D., Sanz, R. \& Batallan F. (2004) "Magnetic properties of densely packed arrays of Ni nanowires as a function of their diameter and lattice parameter" J. Appl. Phys., 95 (11), 6642.

[53] Vázquez, M., Hernández-Vélez, M., Pirota, K., Asenjo, A., Navas, D., Velázquez, J., Vargas, P. \& Ramos C. (2004) "Arrays of $\mathrm{Ni}$ nanowires in alumina membranes: magnetic properties and spatial ordering" The European Physical Journal B, 40 (4), 489.

[54] Vila L., George J. M., Faini G., Popa A., Ebels U., Ounadjela K. \& Piraux L. (2002) "Transport and magnetic properties of isolated cobalt nanowires" IEEE Trans. Mag., 38 (5), 2577.

[55] Vila, L., Piraux, L., George, J. M. \& Faini, G. (2002) "Multiprobe magnetoresistance measurements on isolated magnetic nanowires" Appl. Phys. Lett., 80 (20), 3805.

[56] Volkert, C. A., Minor, A. M. \& Editors, G. (2007) "Focused ion beam micromachining" MIRS Bulletin, 32, 389.

[57] Wernsdorfer, W., Doudin, B., Mailly, D., Hasselbach, K., Benoit, A, Meier, J., Ansermet, J.-Ph. \& Barbara B. (1996) "Nucleation of magnetization reversal in individual nanosized nickel wires" Phys. Rev. Lett., 77 (9), 1873.

[58] Yamazaki, K. (2008) "Nanofabrication Fundamentals and Applications" World Scientific Publishing Co. Pte. Ltd.

[59] Yoo, B., Rheem, Y., Beyermann, W. P. \& Myung, N. V. (2006) "Magnetically assembled $30 \mathrm{~nm}$ diameter nickel nanowire with ferromagnetic electrodes" Nanotech., 17 (10), 2512. 